\title{
Irreducible antifield formalism for reducible constrained Hamiltonian systems
}

\author{
C. Bizdadea*and S. O. Saliu ${ }^{\dagger}$ \\ Department of Physics, University of Craiova \\ 13 A.I. Cuza Str., Craiova RO-1100, Romania
}

December 3, 2018

\begin{abstract}
Reducible constrained Hamiltonian systems are quantized accordingly an irreducible BRST manner. Our procedure is based on the construction of an irreducible theory which is physically equivalent with the original one. The equivalence between the two systems makes legitimate the substitution of the BRST quantization for the reducible theory by that of the irreducible system. The general formalism is illustrated in detail on a model involving abelian one- and two-form gauge fields.
\end{abstract}

PACS number: 11.10.Ef

\section{Introduction}

It is well-known that there are two BRST approaches to the quantization of arbitrary gauge theories. One of them is based on the Lagrangian formalism [1]- 6] (known as the antifield formalism), while the other is dealing with Hamiltonian aspects [6]-[11]. Both formulations can be applied to irreducible, as well as reducible gauge systems. For reducible theories, it is

*e-mail address: bizdadea@hotmail.com

†e-mail address: osaliu@central.ucv.ro 
necessary to introduce ghosts of ghosts and their antifields in order to ensure the nilpotency of the BRST symmetry. The antifield treatment was extended to constrained Hamiltonian systems [12]- [13], allowing therefore a clearer connection between the Lagrangian and the Hamiltonian BRST symmetries.

In this paper we give a consistent procedure for quantizing reducible Hamiltonian systems with first-class constraints following an irreducible BRST mechanism. Although the idea of replacing a redundant set of first-class constraints by an irreducible one in a larger phase-space is known [6], [14], it has been neither consistently developed nor applied so far to the quantization of reducible gauge theories. Starting with a finite-stage reducible Hamiltonian first-class system, we perform the following steps: (i) we transform the original reducible theory into an irreducible one in a manner that allows the substitution of the BRST quantization of the reducible system by that of the irreducible theory, and (ii) we quantize the extended action of the irreducible system accordingly the antifield-BRST formalism. In consequence, the ghosts of ghosts, as well as their antifields do not appear within our formalism. By virtue of this, our method puts on equal footing the reducible and irreducible constrained Hamiltonian systems from the BRST formalism point of view. As far as we know, such an approach has not been previously published, hence our paper establishes a new result.

The paper is organized in six sections. Section 2 is dealing with enlarging the initial phase-space of an arbitrary first-stage reducible Hamiltonian system by adding some supplementary canonical pairs, and with further constructing an irreducible set of first-class constraints. The irreducible set is derived in a way that ensures the equivalence with the starting first-class set. In Section 3 we establish the physical equivalence between the reducible and irreducible systems. In this light, the physical observables and also the number of physical degrees of freedom associated with both theories are shown to coincide. The physical equivalence allows the replacement of the reducible BRST quantization by the irreducible one. The quantization of the resulting irreducible first-class Hamiltonian system is then performed on behalf of an appropriate gauge-fixing fermion. Section 4 exposes the generalization of our results from the previous sections to finite-stage reducible first-class constraints. In Section 5 we exemplify the general theory in the case of a reducible model describing the Stueckelberg coupling between abelian oneand two-form gauge fields. Section 6 ends the paper with some conclusions. 


\section{First-stage reducible Hamiltonian theories}

In this section we show how one can construct a set of irreducible first-class constraints starting from a first-stage reducible one. We begin with a system described by $N$ canonical pairs $\left(q^{i}, p_{i}\right)$, subject to the first-class constraints

$$
G_{a_{0}}(q, p) \approx 0, a_{0}=1, \ldots, M_{0}
$$

which are assumed to be first-stage reducible

$$
Z_{a_{1}}^{a_{0}} G_{a_{0}}=0, a_{1}=1, \ldots, M_{1}
$$

and suppose that there are no second-class constraints in the theory. In (2) we used the strong equality because one can always define the first-stage reducibility functions such that to have off-shell reducibility. For the sake of simplicity, we assume that $\left(q^{i}, p_{i}\right)$ are bosonic, but the results can be extended to fermions by introducing some appropriate phases. We denote the first-class Hamiltonian by $H$, such that the gauge algebra is expressed by $\left[G_{a_{0}}, G_{b_{0}}\right]=C^{c_{0}}{ }_{a_{0} b_{0}} G_{c_{0}},\left[H, G_{a_{0}}\right]=V_{a_{0}}^{b_{0}} G_{b_{0}}$. Relations (2) indicate that the functions $G_{a_{0}}$ are not all independent. Under these circumstances, we locally

split these functions within the independent and dependent components, $G_{\bar{a}_{0}}$, respectively, $G_{a_{1}}$

$$
G_{a_{0}}=\left(\begin{array}{c}
G_{\bar{a}_{0}} \\
G_{a_{1}}
\end{array}\right), \bar{a}_{0}=1, \ldots, M_{0}-M_{1}
$$

with

$$
G_{a_{1}}=M_{a_{1}}^{\bar{a}_{0}} G_{\bar{a}_{0}}
$$

for some functions $M_{a_{1}}^{\bar{a}_{0}}$, such that $G_{\bar{a}_{0}} \approx 0 \Rightarrow G_{a_{1}} \approx 0$. All that is required is to choose the functions $G_{a_{0}}$ in such a way that the split can be achieved in principle. With the help of (幽) we solve (2) with respect to $Z_{a_{1}}^{a_{0}}$. Accordingly, we find

$$
Z_{b_{1}}^{a_{0}}=\left(M_{b_{1}}^{\bar{a}_{0}},-\delta_{b_{1}}^{a_{1}}\right) .
$$

Next, we perform a transformation

$$
G_{a_{0}} \rightarrow \tilde{G}_{a_{0}}=\left(\begin{array}{c}
G_{\bar{a}_{0}} \\
\mathbf{0}
\end{array}\right)
$$


with the help of an invertible matrix $M_{a_{0}}^{b_{0}}$,

$$
\tilde{G}_{a_{0}}=M_{a_{0}}^{b_{0}} G_{b_{0}},
$$

such that $\tilde{G}_{a_{0}} \approx 0 \Leftrightarrow G_{a_{0}} \approx 0$. This matrix allows the representation

$$
M_{a_{0}}^{b_{0}}=\left(\begin{array}{cc}
\delta_{\bar{a}_{0}}^{\bar{b}_{0}} & 0 \\
M_{a_{1}}^{\bar{b}_{0}} & -\delta_{a_{1}}^{b_{1}}
\end{array}\right),
$$

while its inverse coincides with itself

$$
\bar{M}_{a_{0}}^{b_{0}}=M_{a_{0}}^{b_{0}} .
$$

If one inverses (7), one gets

$$
G_{a_{0}}=M_{a_{0}}^{b_{0}} \tilde{G}_{b_{0}},
$$

so, on account of (10) and (21), we consequently find

$$
Z_{b_{1}}^{a_{0}} G_{a_{0}}=Z_{b_{1}}^{a_{0}} M_{a_{0}}^{b_{0}} \tilde{G}_{b_{0}}=0 .
$$

In this way, we can regard

$$
\tilde{Z}_{c_{1}}^{b_{0}}=Z_{c_{1}}^{a_{0}} M_{a_{0}}^{b_{0}}
$$

as the reducibility functions of $\tilde{G}_{b_{0}}$. Using (5) and (8) it follows that $\tilde{Z}_{c_{1}}^{b_{0}}$ is given by

$$
\tilde{Z}_{c_{1}}^{b_{0}}=\left(\mathbf{0}, \delta_{c_{1}}^{b_{1}}\right) \text {. }
$$

If one splits the free index in (12) like $b_{0}=\left(\bar{b}_{0}, b_{1}\right)$ and uses (13), one derives (for $b_{0} \rightarrow b_{1}$ )

$$
Z_{c_{1}}^{a_{0}} M_{a_{0}}^{b_{1}}=\delta_{c_{1}}^{b_{1}}
$$

hence

$$
\operatorname{rank}\left(Z_{c_{1}}^{a_{0}} M_{a_{0}}^{b_{1}}\right)=M_{1},
$$

where

$$
M_{a_{0}}^{b_{1}}=\left(\begin{array}{c}
0 \\
-\delta_{a_{1}}^{b_{1}}
\end{array}\right) .
$$


Next, we transform the reducible constraints (四) into some irreducible ones. In this respect, we introduce a canonical pair $\left(y^{a_{1}}, \pi_{a_{1}}\right)$ associated with every (free index of) relation (2), which we impose to be constrained by

$$
\pi_{a_{1}} \approx 0
$$

Obviously, the constraints (1) and (17) are first-class and reducible. The theory based on these constraints is physically equivalent with that based only on the constraints (11) as the two systems display the same number of physical degrees of freedom, and, moreover, it can be shown that they describe the same physical observables. Indeed, if $f$ denotes an observable of the theory with the constraints (1) and (17), then it is also an observable of the original one. The last statement arises in a simple manner by writing down the equations satisfied by $f$, namely,

$$
\left[f, G_{a_{0}}\right] \approx 0,\left[f, \pi_{a_{1}}\right] \approx 0 .
$$

The equations (18) show that $f$ does not depend (at least weakly) on $y^{a_{1}}$, and, in addition, that the observables associated with this theory fulfill $\left[f, G_{a_{0}}\right] \approx 0$, which are nothing but the equations verified by the observables corresponding to the original system. The converse is also valid, i.e., any observable of the original theory satisfies $(18)$ because it does not depend on $\left(y^{a_{1}}, \pi_{a_{1}}\right)$ and checks by definition $\left[f, G_{a_{0}}\right] \approx 0$.

Dropping out the trivial part of (6), we construct the irreducible first-class constraints

$$
\tilde{\gamma}_{a_{0}}=\left(\begin{array}{c}
G_{\bar{a}_{0}} \\
\pi_{a_{1}}
\end{array}\right) \approx 0
$$

such that the momenta $\pi_{a_{1}}$ replace the dependent constraint functions. With the help of (6) and (16), the constraints (19) can be put under the form

$$
\tilde{\gamma}_{a_{0}}=\tilde{G}_{a_{0}}-M_{a_{0}}^{b_{1}} \pi_{b_{1}} \approx 0 .
$$

Now, we pass from (20) to the equivalent set of first-class constraints

$$
\gamma_{a_{0}}=M_{a_{0}}^{b_{0}} \tilde{\gamma}_{b_{0}}
$$

with $M_{a_{0}}^{b_{0}}$ the matrix (8). Making the notations $A_{a_{0}}^{b_{1}}=-M_{a_{0}}^{b_{0}} M_{b_{0}}^{b_{1}}$ and using (10), we find from (21) the first-class constraints

$$
\gamma_{a_{0}} \equiv G_{a_{0}}+A_{a_{0}}^{b_{1}} \pi_{b_{1}} \approx 0 .
$$


The matrix $A_{a_{0}}^{b_{1}}$ also verifies (14). Indeed, we have that

$$
Z_{c_{1}}^{a_{0}} A_{a_{0}}^{b_{1}}=-Z_{c_{1}}^{a_{0}} M_{a_{0}}^{b_{0}} M_{b_{0}}^{b_{1}}=\tilde{Z}_{c_{1}}^{b_{0}} M_{b_{0}}^{b_{1}}=\delta_{c_{1}}^{b_{1}} .
$$

However, from practical reasons it is useful to weaken the condition (23) by taking $A_{a_{0}}^{b_{1}}$ such that $Z_{c_{1}}^{a_{0}} A_{a_{0}}^{b_{1}}=D_{c_{1}}^{b_{1}}$ is invertible, i.e.,

$$
\operatorname{rank}\left(D_{c_{1}}^{b_{1}}\right)=M_{1} \text {. }
$$

We employ this choice throughout the paper. Moreover, the first-class constraints (22) are irreducible. Indeed, we have that $Z_{a_{1}}^{a_{0}} \gamma_{a_{0}}=D_{a_{1}}^{b_{1}} \pi_{b_{1}}$ is non-vanishing due to (24).

Within the above discussion we supposed that the split of the reducible constraints into independent and dependent ones can be done in principle, this assumption being useful for some technical purposes. Indeed, the split form of the original constraints represents an intermediate step in finally reaching the irreducible constraints (22) where the initial constraint functions appear in a covariant (not split) form. The derivation of the constraints (22) based on the above split is still useful in order to evidence the introduction of the intermediate reducible system possessing the constraints (1) and (17), which subsequently emphasizes in a suggestive manner how the dependent constraints can be replaced by some new degrees of freedom ensuring the irreducibility. However, the separation of the reducible constraints can spoil the covariance or destroy the locality of those relations where it is manifest. In fact, the split hypothesis is not crucial in arriving at (22) and can be replaced by homological arguments, as follows. It is well-known that the BRST symmetry $s_{R}$ associated with a Hamiltonian reducible theory contains two basic differentials

$$
s_{R}=\delta_{R}+D_{R}+\cdots,
$$

where $\delta_{R}$ denotes the Koszul-Tate differential and $D_{R}$ stands for a model of longitudinal derivative along the gauge orbits. In the case of first-stage reducible systems, the action of $\delta_{R}$ on the original phase-space variables and on the generators $\left(\mathcal{P}_{a_{0}}, \mathcal{P}_{a_{1}}\right)$ in the Koszul-Tate complex reads as

$$
\begin{gathered}
\delta_{R} q^{i}=0, \delta_{R} p_{i}=0, \\
\delta_{R} \mathcal{P}_{a_{0}}=-G_{a_{0}},
\end{gathered}
$$




$$
\delta_{R} \mathcal{P}_{a_{1}}=-Z_{a_{1}}^{a_{0}} \mathcal{P}_{a_{0}}
$$

with $\mathcal{P}_{a_{0}}$ and $\mathcal{P}_{a_{1}}$ of antighost number one, respectively, two. The antighosts $\mathcal{P}_{a_{1}}$ are required in order to kill the antighost number one non-trivial co-cycles

$$
\rho_{a_{1}} \equiv Z_{a_{1}}^{a_{0}} \mathcal{P}_{a_{0}}
$$

in the homology of $\delta_{R}$. The idea with the help of which we can recover (22) is to redefine the antighosts $\mathcal{P}_{a_{0}}$ such that the non-trivial co-cycles of the type (29) vanish identically. If we succeed in doing this, the co-cycles (29) do not appear anymore, hence the antighosts $\mathcal{P}_{a_{1}}$ are no longer necessary such that the theory becomes indeed irreducible. In this light, we perform the transformation

$$
\mathcal{P}_{a_{0}} \rightarrow \tilde{\mathcal{P}}_{a_{0}}=D_{a_{0}}^{b_{0}} \mathcal{P}_{b_{0}}
$$

where $D_{a_{0}}^{b_{0}}$ is chosen such that

$$
Z_{a_{1}}^{a_{0}} D_{a_{0}}^{b_{0}}=0, D_{a_{0}}^{b_{0}} G_{b_{0}}=G_{a_{0}}
$$

From (27) and (30 31) we obtain that

$$
\delta \tilde{\mathcal{P}}_{a_{0}}=-G_{a_{0}}
$$

which subsequently leads to

$$
\delta\left(Z_{a_{1}}^{a_{0}} \tilde{\mathcal{P}}_{a_{0}}\right)=0
$$

but with

$$
Z_{a_{1}}^{a_{0}} \tilde{\mathcal{P}}_{a_{0}} \equiv 0
$$

In (32 33) we redenoted $\delta_{R}$ by $\delta$ in order to outline that the new theory is irreducible. If we take

$$
D_{a_{0}}^{b_{0}}=\delta_{a_{0}}^{b_{0}}-Z_{a_{1}}^{b_{0}} \bar{D}_{b_{1}}^{a_{1}} A_{a_{0}}^{b_{1}}
$$

where $\bar{D}_{b_{1}}^{a_{1}}$ is the inverse of $D_{b_{1}}^{a_{1}}$, the equations (31) are clearly satisfied. Substituting (35) in (32) we find that

$$
\delta\left(\mathcal{P}_{a_{0}}-Z_{a_{1}}^{b_{0}} \bar{D}_{b_{1}}^{a_{1}} A_{a_{0}}^{b_{1}} \mathcal{P}_{b_{0}}\right)=-G_{a_{0}}
$$


As the co-cycles (34) vanish identically it results that (32) or (36) can be precisely associated with an irreducible system. In order to derive the form of the irreducible constraints we consider the new canonical pairs $\left(y^{a_{1}}, \pi_{a_{1}}\right)$, with $\pi_{a_{1}}$ the non-trivial solutions of the equations

$$
D_{a_{1}}^{b_{1}} \pi_{b_{1}}=\delta\left(-Z_{a_{1}}^{b_{0}} \mathcal{P}_{b_{0}}\right)
$$

The equations (37) may have trivial, as well as non-trivial solutions. Initially, we notice that $\pi_{b_{1}}=0$ (trivial solutions) if and only if $\delta\left(-Z_{a_{1}}^{b_{0}} \mathcal{P}_{b_{0}}\right)=0$. This case corresponds to the reducible theory with the constraints (1) and (17) (in this situation we obtain the co-cycles (29)). The non-trivial solutions $\pi_{b_{1}} \neq 0$ appear if and only if $\delta\left(-Z_{a_{1}}^{b_{0}} \mathcal{P}_{b_{0}}\right) \neq 0$ (the quantities (29) are no longer co-cycles), hence if and only if the theory is irreducible. While within the split context the momenta $\pi_{b_{1}}$ replace the dependent constraint functions, in the homological approach they enforce the removal of the co-cycles (29). Expressing $\pi_{b_{1}}$ from (37) (in the irreducible case $\pi_{b_{1}} \neq 0$ )

$$
\pi_{b_{1}}=\delta\left(-Z_{a_{1}}^{b_{0}} \bar{D}_{b_{1}}^{a_{1}} \mathcal{P}_{b_{0}}\right),
$$

and replacing this result in (36) we get the relations

$$
\delta \mathcal{P}_{a_{0}}=-G_{a_{0}}-A_{a_{0}}^{b_{1}} \pi_{b_{1}} \equiv-\gamma_{a_{0}} .
$$

The last formulas are nothing but the definitions of $\delta$ on the antighost number one antighosts $\mathcal{P}_{a_{0}}$, that are attached to the irreducible system having the constraints (22). In conclusion, the first-class constraints (22) can be derived by requiring that the non-trivial co-cycles of the type (29) vanish identically under the redefinitions (30). For instance, in the case of free abelian two-form gauge fields the reducible first-class constraints read as

$$
G_{i}^{(2)} \equiv-2 \partial^{j} \pi_{j i} \approx 0 \text {. }
$$

The model is first-stage redundant, namely, $Z^{i} G_{i}^{(2)}=0$, with $Z^{i} \equiv \partial^{i}$. The actions of the reducible Koszul-Tate differential on the antighost number one antighosts $\mathcal{P}_{i}$ are given by

$$
\delta_{R} \mathcal{P}_{i}=2 \partial^{j} \pi_{j i}
$$

Redefining $\mathcal{P}_{i}$ such that

$$
\mathcal{P}_{i} \rightarrow \widetilde{\mathcal{P}}_{i}=\left(\delta_{i}{ }^{j}-\frac{\partial_{i} \partial^{j}}{\triangle}\right) \mathcal{P}_{j} \equiv D_{i}{ }^{j} \mathcal{P}_{j},
$$


from (41) we find

$$
\delta\left(\mathcal{P}_{i}-\frac{\partial_{i} \partial^{j}}{\triangle} \mathcal{P}_{j}\right)=2 \partial^{j} \pi_{j i},
$$

where $\triangle=\partial_{k} \partial^{k}$. Introducing the canonical pair $(\varphi, \pi)$ playing the role of the variables $\left(y^{a_{1}}, \pi_{a_{1}}\right)$ and taking $D_{a_{1}}^{b_{1}}$ to be $-\triangle$, the equations (37) become

$$
\triangle \pi=\delta\left(\partial^{k} \mathcal{P}_{k}\right),
$$

so

$$
\pi=\delta\left(\frac{\partial^{k}}{\triangle} \mathcal{P}_{k}\right) .
$$

Substituting (45) in (43), we find the relations

$$
\delta \mathcal{P}_{i}=2 \partial^{j} \pi_{j i}+\partial_{i} \pi
$$

In this way the relations (46) emphasize the irreducible first-class constraints

$$
\gamma_{i} \equiv-2 \partial^{j} \pi_{j i}-\partial_{i} \pi \approx 0,
$$

which appear for instance in the example from Section 5 in the limit $M=0$ and in the absence of the fields $H^{\mu}$ (see the first relations in formula (133)).

Now we can show that the constraints (11) and (17) are equivalent with (22), i.e.,

$$
\gamma_{a_{0}} \approx 0 \Leftrightarrow G_{a_{0}} \approx 0, \pi_{a_{1}} \approx 0 .
$$

It is simply to see that if (11) and (17) hold, then the constraints (22) also hold. The converse is valid, too. Indeed, we will see that

$$
\gamma_{a_{0}} \approx 0 \Rightarrow G_{a_{0}} \approx 0, \pi_{a_{1}} \approx 0
$$

This can be shown as follows. First, we apply $Z_{c_{1}}^{a_{0}}$ on (22), which then yields

$$
\bar{D}_{a_{1}}^{c_{1}} Z_{c_{1}}^{a_{0}} \gamma_{a_{0}}=\pi_{a_{1}} .
$$

With the help of (22) and (50) we get

$$
\left(\delta_{a_{0}}^{b_{0}}-A_{a_{0}}^{b_{1}} \bar{D}_{b_{1}}^{c_{1}} Z_{c_{1}}^{b_{0}}\right) \gamma_{b_{0}}=G_{a_{0}} .
$$


From (50-51) we reach (49). The Poisson brackets between the irreducible first-class constraints read

$$
\left[\gamma_{a_{0}}, \gamma_{b_{0}}\right]=\bar{C}_{a_{0} b_{0}}^{c_{0}} \gamma_{c_{0}}
$$

where the new structure functions are expressed by

$$
\begin{aligned}
& \bar{C}_{a_{0} b_{0}}^{c_{0}}=C_{a_{0} b_{0}}^{d_{0}}\left(\delta_{d_{0}}^{c_{0}}-A_{d_{0}}^{b_{1}} \bar{D}_{b_{1}}^{c_{1}} Z_{c_{1}}^{c_{0}}\right)+ \\
& \left(\left[G_{a_{0}}, A_{b_{0}}^{b_{1}}\right]+\left[A_{a_{0}}^{b_{1}}, G_{b_{0}}\right]+\left[A_{a_{0}}^{c_{1}}, A_{b_{0}}^{b_{1}}\right] \pi_{b_{1}}\right) \bar{D}_{b_{1}}^{c_{1}} Z_{c_{1}}^{c_{0}} .
\end{aligned}
$$

The first-class Hamiltonian of the new theory can be derived starting from the original one, $H$. Indeed, if we take

$$
H^{\prime}=H+h^{a_{1}}\left(q^{i}, p_{i}\right) \pi_{a_{1}},
$$

with

$$
\left[h^{a_{1}}, G_{a_{0}}\right]=V_{a_{0}}^{b_{0}} A_{b_{0}}^{a_{1}}
$$

we subsequently find

$$
\left[H^{\prime}, \gamma_{a_{0}}\right]=\bar{V}_{a_{0}}^{b_{0}} \gamma_{b_{0}}
$$

where

$$
\bar{V}_{a_{0}}^{b_{0}}=V_{a_{0}}^{b_{0}}+\left(\left[H, A_{a_{0}}^{a_{1}}\right]+\left[h^{b_{1}}, A_{a_{0}}^{a_{1}}\right] \pi_{b_{1}}\right) \bar{D}_{a_{1}}^{c_{1}} Z_{c_{1}}^{b_{0}} .
$$

It is clear that the first-class Hamiltonian (54) is not unique because we can always add to it any combinations of $\gamma_{a_{0}}$ 's with coefficients that are arbitrary functions. The change induced by the modification of the Hamiltonian gives raise to a change in the structure functions (57).

In brief, in this section we constructed an irreducible first-class system associated with the original redundant one, described by the constraints (22) and the first-class Hamiltonian (54), displaying the gauge algebra (52) and (56). The irreducible theory built here will be important by virtue of the subsequent development.

\section{Irreducible quantization of the reducible the- ory}

Now, we show that the reducible, respectively, irreducible theories possess the same classical observables. We start from an observable $F$ of the irreducible 
theory. Accordingly, $F$ should verify the equations

$$
\left[F, \gamma_{a_{0}}\right] \approx 0 .
$$

On account of (22) and (50), from (58) we deduce

$$
\left[F, G_{a_{0}}\right]+\left[F, \pi_{a_{1}}\right] A_{a_{0}}^{a_{1}} \approx 0 .
$$

On the other hand, multiplying (59) with $Z_{b_{1}}^{a_{0}}$ and using (51), we arrive at

$$
\left[F, \pi_{a_{1}}\right] D_{b_{1}}^{a_{1}} \approx\left[F, Z_{b_{1}}^{a_{0}}\right]\left(\delta_{a_{0}}^{b_{0}}-A_{a_{0}}^{c_{1}} \bar{D}_{c_{1}}^{d_{1}} Z_{d_{1}}^{b_{0}}\right) \gamma_{b_{0}} \approx 0 .
$$

Because $D_{b_{1}}^{a_{1}}$ has maximal rank (see (24)), from (60) we infer

$$
\left[F, \pi_{a_{1}}\right] \approx 0,
$$

such that

$$
\left[F, \gamma_{a_{0}}\right] \approx 0 \Rightarrow\left[F, G_{a_{0}}\right] \approx 0 .
$$

In conclusion, if $F$ is an observable of the irreducible theory, then it is also an observable of the original reducible one. The converse is valid, too, because any observable of the reducible theory verifies the equations $\left[F, G_{a_{0}}\right] \approx 0$ and does not depend on the newly added canonical variables, such that (58) are indeed satisfied. Thus, both the irreducible and reducible models display the same physical observables. A simple count shows that the numbers of physical degrees of freedom of the reducible, respectively, irreducible theories are both equal to $N-M_{0}+M_{1}$. The last conclusions prove that the original reducible theory is physically equivalent with the irreducible one. This makes permissible the replacement of the BRST quantization for the original redundant system by the BRST quantization of the irreducible theory.

The first attempt at quantizing the irreducible system is to apply the antifield-BRST formalism with respect to its extended action, namely,

$$
S_{0}^{\prime E}\left[q^{i}, p_{i}, y^{a_{1}}, \pi_{a_{1}}, u^{a_{0}}\right]=\int d t\left(\dot{q}^{i} p_{i}+\dot{y}^{a_{1}} \pi_{a_{1}}-H^{\prime}-u^{a_{0}} \gamma_{a_{0}}\right) .
$$

Action (63) is invariant under the gauge transformations

$$
\delta_{\epsilon} F=\left[F, \gamma_{a_{0}}\right] \epsilon^{a_{0}}, \delta_{\epsilon} u^{a_{0}}=\dot{\epsilon}^{a_{0}}-\bar{V}_{b_{0}}{ }^{a_{0}} \epsilon^{b_{0}}-\bar{C}_{b_{0} c_{0}}^{a_{0}} u^{b_{0}} \epsilon^{c_{0}},
$$


with $\epsilon^{a_{0}}$ the gauge parameters associated with the irreducible constraints (22). In the absence of the newly introduced variables, the extended action (63) together with its gauge transformations, (64), should reduce to those from the reducible case. The gauge transformations of the Lagrange multipliers from (64) do not lead to the corresponding transformations from the reducible situation because the terms $-Z_{a_{1}}^{a_{0}} \epsilon^{a_{1}}$ are missing. The gauge parameters $\epsilon^{a_{1}}$, which were attached to the first-stage reducibility functions, are absent within the irreducible approach. In order to restore these terms, it is necessary to further enlarge the phase-space by adding some supplementary canonical pairs $\left(z_{1}^{a_{1}}, p_{1 a_{1}}\right),\left(z_{2}^{a_{1}}, p_{2 a_{1}}\right)$, subject to the constraints

$$
-p_{1 a_{1}} \approx 0, p_{2 a_{1}} \approx 0
$$

Obviously, (22) and (65) are still first-class and irreducible. Adding to the first set of constraints from (65) a combination of first-class constraints (see (50)), we obtain the equivalent first-class set

$$
\gamma_{a_{1}} \equiv \pi_{a_{1}}-p_{1 a_{1}} \approx 0, \gamma_{a_{1}}^{\prime} \equiv p_{2 a_{1}} \approx 0 .
$$

The additional first-class constraints do not afflict the number of physical degrees of freedom of the former irreducible system. At the same time, the above established equivalence between the physical observables respectively associated with the reducible and irreducible theories remains valid. This is because an observable $F$ of the last irreducible model must check, beside (58), the equations

$$
\left[F, \gamma_{a_{1}}\right] \approx 0,\left[F, \gamma_{a_{1}}^{\prime}\right] \approx 0
$$

On account of (61) relations (67) indicate that in addition to the prior conditions $F$ does not depend (at least weakly) on the last added canonical pairs. As it will be seen below, the constraints (66) will imply the presence of the terms $-Z_{a_{1}}^{a_{0}} \epsilon^{a_{1}}$ within the gauge transformations of the Lagrange multipliers $u^{a_{0}}$. In this sense, the constraints (66) play in a certain way the role of the original reducibility relations. The first-class Hamiltonian with respect to the first-class set (22) and (66) can be taken as

$$
H_{0}=H^{\prime}+z_{2}^{a_{1}} Z_{a_{1}}^{a_{0}} \gamma_{a_{0}}+y^{a_{1}} \gamma_{a_{1}}^{\prime},
$$

such that the new irreducible gauge algebra reads

$$
\left[\gamma_{a_{0}}, \gamma_{b_{0}}\right]=\bar{C}_{a_{0} b_{0}}^{c_{0}} \gamma_{c_{0}},\left[\gamma_{a_{0}}, \gamma_{a_{1}}\right]=0,\left[\gamma_{a_{0}}, \gamma_{a_{1}}^{\prime}\right]=0,
$$




$$
\begin{gathered}
{\left[\gamma_{a_{1}}, \gamma_{b_{1}}\right]=0,\left[\gamma_{a_{1}}, \gamma_{b_{1}}^{\prime}\right]=0,\left[\gamma_{a_{1}}^{\prime}, \gamma_{b_{1}}^{\prime}\right]=0} \\
{\left[H_{0}, \gamma_{a_{0}}\right]=\tilde{V}_{a_{0}}^{b_{0}} \gamma_{b_{0}}+A_{a_{0}}^{b_{1}} \gamma_{b_{1}}^{\prime},\left[H_{0}, \gamma_{a_{1}}\right]=\gamma_{a_{1}}^{\prime},\left[H_{0}, \gamma_{a_{1}}^{\prime}\right]=Z_{a_{1}}^{a_{0}} \gamma_{a_{0}} .}
\end{gathered}
$$

Simple calculations show that the functions $\tilde{V}_{a_{0}}^{b_{0}}$ from (71) are of the form

$$
\tilde{V}_{a_{0}}^{b_{0}}=\bar{V}_{a_{0}}^{b_{0}}+z_{2}^{a_{1}}\left(\mu_{a_{0} a_{1}}^{b_{0} c_{0}} G_{c_{0}}+\lambda_{a_{0} a_{1}}^{b_{1}} Z_{b_{1}}^{b_{0}}+\left[Z_{a_{1}}^{b_{0}}, A_{a_{0}}^{b_{1}}\right] \pi_{b_{1}}\right)
$$

with $\mu_{a_{0} a_{1}}^{b_{0} c_{0}}$ and $\lambda_{a_{0} a_{1}}^{b_{1}}$ appearing in

$$
\left[Z_{a_{1}}^{c_{0}}, G_{d_{0}}\right]=-Z_{a_{1}}^{a_{0}} C_{a_{0} d_{0}}^{c_{0}}+\mu_{d_{0} a_{1}}^{c_{0} b_{0}} G_{b_{0}}+\lambda_{d_{0} a_{1}}^{b_{1}} Z_{b_{1}}^{c_{0}}
$$

and $\mu_{a_{0} a_{1}}^{b_{0} c_{0}}$ antisymmetric in the upper indices, i.e., $\mu_{a_{0} a_{1}}^{b_{0} c_{0}}=-\mu_{a_{0} a_{1}}^{c_{0} b_{0}}$. The relations (73) can be inferred by taking the Poisson brackets between (2) and $G_{d_{0}}$, which leads to

$$
\left(Z_{a_{1}}^{a_{0}} C_{a_{0} d_{0}}^{c_{0}}+\left[Z_{a_{1}}^{c_{0}}, G_{d_{0}}\right]\right) G_{c_{0}}=0 .
$$

From (74) and (2) it follows directly (73). We outline that the functions $\bar{C}^{c_{0}}{ }_{a_{0} b_{0}}$ and $\tilde{V}_{a_{0}}^{b_{0}}$ encode the reducible structure within the irreducible theory. The first-class Hamiltonian (68) is unique up to a combination in terms of the functions $\gamma_{a_{0}}, \gamma_{a_{1}}$ and $\gamma_{a_{1}}^{\prime}$. The change of $H_{0}$ consequently implies the change of the structure functions from (71).

The extended action describing the new irreducible theory

$$
\begin{aligned}
& S_{0}^{E}\left[q^{i}, p_{i}, y^{a_{1}}, \pi_{a_{1}}, z_{1}^{a_{1}}, p_{1 a_{1}}, z_{2}^{a_{1}}, p_{2 a_{1}}, u^{a_{0}}, u^{a_{1}}, v^{a_{1}}\right]=\int d t\left(\dot{q}^{i} p_{i}+\right. \\
& \left.\dot{y}^{a_{1}} \pi_{a_{1}}+\dot{z}_{1}^{a_{1}} p_{1 a_{1}}+\dot{z}_{2}^{a_{1}} p_{2 a_{1}}-H_{0}-u^{a_{0}} \gamma_{a_{0}}-u^{a_{1}} \gamma_{a_{1}}-v^{a_{1}} \gamma_{a_{1}}^{\prime}\right)
\end{aligned}
$$

is invariant under the gauge transformations

$$
\begin{gathered}
\delta_{\epsilon} F=\left[F, \gamma_{a_{0}}\right] \epsilon^{a_{0}}+\left[F, \gamma_{a_{1}}\right] \epsilon_{1}^{a_{1}}+\left[F, \gamma_{a_{1}}^{\prime}\right] \epsilon_{2}^{a_{1}}, \\
\delta_{\epsilon} u^{a_{0}}=\dot{\epsilon}^{a_{0}}-\tilde{V}_{b_{0}}^{a_{0}} \epsilon^{b_{0}}-\bar{C}_{b_{0} c_{0}}^{a_{0}} u^{b_{0}} \epsilon^{c_{0}}-Z_{a_{1}}^{a_{0}} \epsilon_{2}^{a_{1}}, \\
\delta_{\epsilon} u^{a_{1}}=\dot{\epsilon}_{1}^{a_{1}}, \delta_{\epsilon} v^{a_{1}}=\dot{\epsilon}_{2}^{a_{1}}-A_{a_{0}}^{a_{1}} \epsilon^{a_{0}}-\epsilon_{1}^{a_{1}} .
\end{gathered}
$$

We emphasize that in this way the terms $-Z_{a_{1}}^{a_{0}} \epsilon_{2}^{a_{1}}$ are restored within the gauge transformations of the multipliers $u^{a_{0}}$. This is precisely the effect of introducing the supplementary pairs $\left(z_{1}^{a_{1}}, p_{1 a_{1}}\right),\left(z_{2}^{a_{1}}, p_{2 a_{1}}\right)$ subject to the 
constraints (66). If in (75, 78 ) we discard all the newly introduced canonical pairs, we get the extended action and the gauge transformations from the initial redundant case.

With these elements at hand, it appears clearly that we can substitute the quantization of the initial theory by the quantization of the last irreducible system. In the sequel we perform the antifield-BRST quantization with respect to the action (75). To this end, we introduce the ghosts

$$
\left(\eta^{a_{0}}, \eta_{1}^{a_{1}}, \eta_{2}^{a_{1}}\right)
$$

and also the antifields

$$
\left(q_{i}^{*}, p^{* i}, y_{a_{1}}^{*}, \pi^{* a_{1}}, z_{1 a_{1}}^{*}, p_{1}^{* a_{1}}, z_{2 a_{1}}^{*}, p_{2}^{* a_{1}}, u_{a_{0}}^{*}, u_{a_{1}}^{*}, v_{a_{1}}^{*}, \eta_{a_{0}}^{*}, \eta_{1 a_{1}}^{*}, \eta_{2 a_{1}}^{*}\right) .
$$

The ghosts have ghost number one, the antifields associated with the variables involved with (75) possess ghost number minus one, while the antifields of the ghosts have ghost number minus two. The solution to the master equation is given by

$$
\begin{aligned}
& S^{E}=S_{0}^{E}+\int d t\left(q_{i}^{*}\left[q^{i}, \gamma_{a_{0}}\right] \eta^{a_{0}}+p^{* i}\left[p_{i}, \gamma_{a_{0}}\right] \eta^{a_{0}}+\right. \\
& y_{a_{1}}^{*}\left(A_{a_{0}}^{a_{1}} \eta^{a_{0}}+\eta_{1}^{a_{1}}\right)-z_{1 a_{1}}^{*} \eta_{1}^{a_{1}}+z_{2 a_{1}}^{*} \eta_{2}^{a_{1}}+ \\
& u_{a_{0}}^{*}\left(\dot{\eta}^{a_{0}}-\tilde{V}_{b_{0}}^{a_{0}} \eta^{b_{0}}-\bar{C}_{b_{0} c_{0}}^{a_{0}} u^{b_{0}} \eta^{c_{0}}-Z_{a_{1}}^{a_{0}} \eta_{2}^{a_{1}}\right)+ \\
& \left.u_{a_{1}}^{*} \dot{\eta}_{1}^{a_{1}}+v_{a_{1}}^{*}\left(\dot{\eta}_{2}^{a_{1}}-A_{a_{0}}^{a_{1}} \eta^{a_{0}}-\eta_{1}^{a_{1}}\right)-\frac{1}{2} \eta_{a_{0}}^{*} \bar{C}_{b_{0} c_{0}}^{a_{0}} \eta^{b_{0}} \eta^{c_{0}}+\cdots\right) .
\end{aligned}
$$

In order to derive a gauge-fixed action, it is necessary to fix the gauge. In this respect, it is useful to take a gauge-fixing fermion

$$
\psi=\psi\left[q^{i}, p_{i}, y^{a_{1}}, \pi_{a_{1}}, z_{1}^{a_{1}}, p_{1 a_{1}}, z_{2}^{a_{1}}, p_{2 a_{1}}, \eta^{a_{0}}, \eta_{1}^{a_{1}}, \eta_{2}^{a_{1}}, u_{a_{0}}^{*}, u_{a_{1}}^{*}, v_{a_{1}}^{*}\right],
$$

implementing some irreducible gauge conditions, with the help of which we eliminate all the antifields excepting $u_{a_{0}}^{*}, u_{a_{1}}^{*}, v_{a_{1}}^{*}$, that are maintained in favour of their fields. The possibility to build some irreducible gauge conditions is easier on behalf of the newly added canonical pairs, which play at this level the same role like the auxiliary variables from the reducible approach. We can put the gauge-fixed action under a form displaying a more direct link with the Hamiltonian BRST quantization of the irreducible system following the procedure exposed in [12]-13]. In this light, we declare 
the variables $\left(\eta^{a_{0}}, u_{a_{0}}^{*}\right),\left(\eta_{1}^{a_{1}}, u_{a_{1}}^{*}\right),\left(\eta_{2}^{a_{1}}, v_{a_{1}}^{*}\right)$ respectively conjugated in the Poisson bracket

$$
\left[u_{a_{0}}^{*}, \eta^{b_{0}}\right]=-\delta_{a_{0}}^{b_{0}},\left[u_{a_{1}}^{*}, \eta_{1}^{b_{1}}\right]=-\delta_{a_{1}}^{b_{1}},\left[v_{a_{1}}^{*}, \eta_{2}^{b_{1}}\right]=-\delta_{a_{1}}^{b_{1}},
$$

and regard the antifields like the momenta associated with the ghosts. Under these circumstances, the gauge-fixed action corresponding to (81) reads as

$$
\begin{aligned}
& S_{\psi}=\int d t\left(\dot{q}^{i} p_{i}+\dot{y}^{a_{1}} \pi_{a_{1}}+\dot{z}_{1}^{a_{1}} p_{1 a_{1}}+\dot{z}_{2}^{a_{1}} p_{2 a_{1}}+\right. \\
& \left.u_{a_{0}}^{*} \dot{\eta}^{a_{0}}+u_{a_{1}}^{*} \dot{\eta}_{1}^{a_{1}}+v_{a_{1}}^{*} \dot{\eta}_{2}^{a_{1}}-H_{B}+[\psi, \Omega]\right),
\end{aligned}
$$

where the BRST charge, respectively, the BRST-extension of the first-class Hamiltonian start like

$$
\begin{gathered}
\Omega=\gamma_{a_{0}} \eta^{a_{0}}+\gamma_{a_{1}} \eta_{1}^{a_{1}}+\gamma_{a_{1}}^{\prime} \eta_{2}^{a_{1}}+\frac{1}{2} u_{a_{0}}^{*} \bar{C}_{b_{0} c_{0}}^{a_{0}} \eta^{b_{0}} \eta^{c_{0}}+\cdots, \\
H_{B}=H_{0}+u_{a_{0}}^{*}\left(\tilde{V}_{b_{0}}^{a_{0}} \eta^{b_{0}}+Z_{a_{1}}^{a_{0}} \eta_{2}^{a_{1}}\right)+v_{a_{1}}^{*}\left(A_{a_{0}}^{a_{1}} \eta^{a_{0}}+\eta_{1}^{a_{1}}\right)+\cdots .
\end{gathered}
$$

This completes our irreducible procedure in the case of first-stage reducible first-class Hamiltonian theories. Until now, we showed how a first-stage reducible first-class Hamiltonian system can be quantized in the framework of the irreducible antifield-BRST formalism, i.e., without introducing ghosts of ghosts.

\section{$4 \quad L$-stage reducible Hamiltonian theories}

In this section we generalize the results from the first-stage case to higherorder-stage reducible systems. If the original Hamiltonian theory is $L$-stage reducible (with finite $L$ ), the construction of the corresponding irreducible system goes along the same line like that from the first-stage case. We assume the reducibility relations

$$
Z_{a_{1}}^{a_{0}} G_{a_{0}}=0, Z_{a_{1}}^{a_{0}} Z_{a_{2}}^{a_{1}}=0, \cdots, Z_{a_{L-1}}^{a_{L-2}} Z_{a_{L}}^{a_{L-1}}=0,
$$

with $a_{k}=1, \cdots, M_{k}$. Next, we introduce the canonical pairs $\left(y^{a_{k}}, \pi_{a_{k}}\right)_{k=1, \cdots, L}$ corresponding to the free indices of the above reducibility relations, and constrain these new variables like

$$
\pi_{a_{k}} \approx 0
$$


Constraints (11) and (88) are first-class and obviously reducible. In a manner similar with that from section 2 , we derive the first-class constraints

$$
\begin{aligned}
& \gamma_{a_{0}} \equiv G_{a_{0}}+A_{a_{0}}^{b_{1}} \pi_{b_{1}} \approx 0 \\
& \gamma_{a_{2 k}} \equiv Z_{a_{2 k}}^{a_{2 k-1}} \pi_{a_{2 k-1}}+A_{a_{2 k}}^{a_{2 k+1}} \pi_{a_{2 k+1}} \approx 0, k=1, \cdots, a, \\
& \bar{\gamma}_{a_{2 k}} \equiv \pi_{a_{2 k}} \approx 0, k=1, \cdots, a,
\end{aligned}
$$

which are equivalent with (1) and (88). Acting like in the first-stage situation, we find that

$$
\pi_{a_{2 k+1}}=m_{a_{2 k+1}}^{b_{2 k}} \gamma_{b_{2 k}}, G_{a_{0}}=m_{a_{0}}^{b_{2 k}} \gamma_{b_{2 k}}, k=0, \cdots, b,
$$

for some appropriate functions $m_{a_{2 k+1}}^{b_{2 k}}$ and $m_{a_{0}}^{b_{2 k}}$, such that the equivalence between (11), (88) and (89 91) is direct. We employed the notations

$$
\begin{gathered}
a=\left\{\begin{array}{c}
\frac{L}{2}, \text { for } L \text { even, } \\
\frac{L-1}{2}, \text { for } L \text { odd },
\end{array}\right. \\
b=\left\{\begin{array}{c}
\frac{L}{2}-1, \text { for } L \text { even, } \\
\frac{L-1}{2}, \text { for } L \text { odd } .
\end{array}\right.
\end{gathered}
$$

In (90) the functions $A_{a_{k}}^{a_{k+1}}$ depend only on $\left(q^{i}, p_{i}\right)$ and possess the property

$$
\operatorname{rank}\left(Z_{a_{k}}^{a_{k-1}} A_{a_{k-1}}^{b_{k}}\right) \approx \sum_{i=k}^{L}(-)^{k+i} M_{i} .
$$

Moreover, the $A_{a_{k-1}}^{b_{k}}$ 's can be taken to satisfy the relations

$$
A_{a_{k-1}}^{b_{k}} A_{b_{k}}^{a_{k+1}}=0
$$

The last relations are based on the fact that we can always choose the $A_{a_{k-1}}^{b_{k}}$ 's proportional with the transposed of $Z_{b_{k}}^{a_{k-1}}$ 's. On account of (96), one finds that the first-class set (89 91) is irreducible. We remark that (91) are irreducible. Thus, it remains to be proved that (89 90) are so. This can be seen by multiplying (89) with $Z_{b_{1}}^{a_{0}}$ and (90) with $Z_{b_{2 k+1}}^{a_{2 k}}$, which induce

$$
Z_{b_{1}}^{a_{0}} \gamma_{a_{0}}=Z_{b_{1}}^{a_{0}} A_{a_{0}}^{c_{1}} \pi_{c_{1}}, Z_{b_{2 k+1}}^{a_{2 k}} \gamma_{a_{2 k}}=Z_{b_{2 k+1}}^{a_{2 k}} A_{a_{2 k}}^{a_{2 k+1}} \pi_{a_{2 k+1}} .
$$


With the help of (97) and (96) we infer that $Z_{b_{1}}^{a_{0}} \gamma_{a_{0}}=0, Z_{b_{2 k+1}}^{a_{2 k}} \gamma_{a_{2 k}}=0$ if and only if

$$
\pi_{a_{2 k+1}}=A_{a_{2 k+1}}^{a_{2 k+2}} \nu_{a_{2 k+2}}, k=0, \cdots, b,
$$

with $\nu_{a_{2 k+2}}$ some functions. Replacing (98) in (89 90) we obtain

$$
G_{a_{0}} \approx 0, Z_{b_{2 k}}^{a_{2 k-1}} A_{a_{2 k-1}}^{a_{2 k}} \nu_{a_{2 k}} \approx 0
$$

which leads, by virtue of (9596), to

$$
\nu_{a_{2 k}} \approx A_{a_{2 k}}^{a_{2 k+1}} \lambda_{a_{2 k+1}}
$$

for some $\lambda_{a_{2 k+1}}$. Substituting (100) in (98) we derive that $(89,90)$ are reducible with the reducibility functions $Z_{b_{2 k+1}}^{a_{2 k}}$ if and only if $\pi_{a_{2 k+1}} \approx 0$. In this situation the constraints (89 90) and (91) are nothing but (11) and (88). Thus, (89 91) are reducible with the reducibility functions $Z_{b_{2 k+1}}^{a_{2 k}}$ if and only if they have the form (11) and (88). On the other hand, if one multiplies (90) by $A_{b_{2 k-1}}^{a_{2 k}}$, one gets

$$
A_{b_{2 k-1}}^{a_{2 k}} \gamma_{a_{2 k}}=A_{b_{2 k-1}}^{a_{2 k}} Z_{a_{2 k}}^{a_{2 k-1}} \pi_{a_{2 k-1}}
$$

due to (96). From (101), it results that $A_{b_{2 k-1}}^{a_{2 k}} \gamma_{a_{2 k}}=0$ if and only if

$$
\pi_{a_{2 k-1}}=Z_{a_{2 k-1}}^{a_{2 k-2}} \mu_{a_{2 k-2}},
$$

for some $\mu_{a_{2 k-2}}$. Inserting (102) in (90) we find

$$
A_{a_{2 k}}^{a_{2 k+1}} Z_{a_{2 k+1}}^{b_{2 k}} \mu_{b_{2 k}} \approx 0
$$

which leads to

$$
\mu_{b_{2 k}} \approx Z_{b_{2 k}}^{a_{2 k-1}} \sigma_{a_{2 k-1}},
$$

for some $\sigma_{a_{2 k-1}}$. Introducing (104) in (102) we conclude that (89 91) are reducible with the reducibility functions $A_{b_{2 k-1}}^{a_{2 k}}$ if and only if they reduce to (11) and (88). In this way, the irreducibility of (89 91) is completely proved.

In the meantime, it is still necessary to add the pairs $\left(z_{1}^{a_{2 k+1}}, p_{1 a_{2 k+1}}\right)$, $\left(z_{2}^{a_{2 k+1}}, p_{2 a_{2 k+1}}\right)$, with $k=0, \cdots, b$. With the help of the last pairs we build the supplementary first-class constraints

$$
\gamma_{a_{2 k+1}} \equiv \pi_{a_{2 k+1}}-p_{1 a_{2 k+1}} \approx 0, \gamma_{a_{2 k+1}}^{\prime} \equiv p_{2 a_{2 k+1}} \approx 0
$$


The equivalence between the observables of the original redundant theory and those of the irreducible system is gained like in the first-stage situation. We illustrate the proof of the equivalence in the case $L$ odd, the other situation being treated in a similar fashion. If $F$ stands for an observable of the irreducible system, the conditions $\left[F, \bar{\gamma}_{a_{2 k}}\right] \approx 0$ indicate that it does not depend, at least weakly, on $y^{a_{2 k}}$. In the meantime, $F$ should verify

$$
\left[F, \gamma_{a_{2 k}}\right] \approx 0, k=0, \cdots, a .
$$

We start from the last relation (106) (for $k=a$ ). On account of (92), we obtain

$$
\left[F, \pi_{a_{L-2}}\right] Z_{a_{L-1}}^{a_{L-2}}+\left[F, \pi_{a_{L}}\right] A_{a_{L-1}}^{a_{L}} \approx 0 .
$$

Multiplying the above equation by $Z_{b_{L}}^{a_{L-1}}$, on behalf of (95), and as $M_{L+1}=0$, we derive

$$
\left[F, \pi_{a_{L}}\right] \approx 0,
$$

such that (107) becomes

$$
\left[F, \pi_{a_{L-2}}\right] Z_{a_{L-1}}^{a_{L-2}} \approx 0 .
$$

Multiplying the next equation from (106) (for $k=a-1$ ) with $Z_{b_{L-2}}^{a_{L-3}}$, we further infer

$$
\left[F, \pi_{a_{L-2}}\right] A_{a_{L-3}}^{a_{L-2}} Z_{b_{L-2}}^{a_{L-3}} \approx 0 .
$$

With the help of (96), from (110) we deduce

$$
\left[F, \pi_{a_{L-2}}\right]=n_{b_{L-1}} A_{a_{L-2}}^{b_{L-1}},
$$

for some functions $n_{b_{L-1}}$. Replacing (111) in (109) it follows that the weak relations $n_{b_{L-1}} A_{a_{L-2}}^{b_{L-1}} Z_{a_{L-1}}^{a_{L-2}} \approx 0$ imply

$$
n_{b_{L-1}} \approx \rho_{b_{L}} A_{b_{L-1}}^{b_{L}},
$$

for some $\rho_{b_{L}}$. Inserting (112) in (111) we get

$$
\left[F, \pi_{a_{L-2}}\right] \approx 0,
$$

due to (96). Reprising the same steps on the remaining equations (106) we consequently arrive to

$$
\left[F, \pi_{a_{L-2 k}}\right] \approx 0,
$$


which lead to

$$
\left[F, G_{a_{0}}\right] \approx 0 .
$$

Moreover, the equations $\left[F, \gamma_{a_{2 k+1}}\right] \approx 0$ and $\left[F, \gamma_{a_{2 k+1}}^{\prime}\right] \approx 0$ express the fact that $F$ does not depend on the $z$ 's. Thus, any observable of the irreducible theory does not involve, at least weakly, the newly introduced variables, and, in addition, it satisfies (115), which are nothing but the equations that should be checked by any observable of the original redundant system, which show that $F$ is also an observable of the reducible theory. Conversely, if $F^{\prime}$ denotes an observable associated with the reducible system, then it is obviously an observable of the irreducible theory.

The first-class Hamiltonian with respect to the irreducible first-class constraints (89 91) and (105) can be taken under the form

$$
\begin{aligned}
& H_{0}^{\prime}=H^{\prime}+\sum_{k=1}^{a} y^{a_{2 k}} \gamma_{a_{2 k}}+\sum_{k=0}^{b} y^{a_{2 k+1}} p_{2 a_{2 k+1}}+ \\
& \sum_{k=0}^{b} z_{2}^{a_{2 k+1}}\left(Z_{a_{2 k+1}}^{a_{2 k}} \gamma_{a_{2 k}}+A_{a_{2 k+1}}^{a_{2 k+2}} \gamma_{a_{2 k+2}}\right),
\end{aligned}
$$

with $H^{\prime}$ given by (54), where we understood the convention $f^{a_{k}}=0$ if $k<0$ or $k>L$. The first-class Hamiltonian (116) is again unique up to adding a combination in the first-class constraint functions.

With all these elements at hand, the quantization of the irreducible theory goes from now on along the standard antifield-BRST rules. The ghost spectrum contains only the ghost number one variables associated with the corresponding constraint functions

$$
\begin{gathered}
\eta^{a_{0}} \leftrightarrow \gamma_{a_{0}}, \eta_{1}^{a_{2 k}} \leftrightarrow \bar{\gamma}_{a_{2 k}}, \eta_{2}^{a_{2 k}} \leftrightarrow \gamma_{a_{2 k}}, k=1, \cdots, a, \\
\eta_{1}^{a_{2 k+1}} \leftrightarrow \gamma_{a_{2 k+1}}, \eta_{2}^{a_{2 k+1}} \leftrightarrow \gamma_{a_{2 k+1}}^{\prime}, k=0, \cdots, b,
\end{gathered}
$$

while the antifield sector is given by

$$
\begin{gathered}
\left(q_{i}^{*}, p^{* i}\right),\left(y_{a_{k}}^{*}, \pi^{* a_{k}}\right)_{k=1, \cdots, L},\left(z_{1 a_{2 k+1}}^{*}, p_{1}^{* a_{2 k+1}}\right)_{k=0, \cdots, b},\left(z_{2 a_{2 k+1}}^{*}, p_{2}^{* a_{2 k+1}}\right)_{k=0, \cdots, b}, \\
u_{a_{0}}^{*},\left(u_{a_{2 k}}^{*}, v_{a_{2 k}}^{*}\right)_{k=1, \cdots, a},\left(u_{a_{2 k+1}}^{*}, v_{a_{2 k+1}}^{*}\right)_{k=0, \cdots, b}, \\
\eta_{a_{0}}^{*},\left(\eta_{1 a_{2 k}}^{*}, \eta_{2 a_{2 k}}^{*}\right)_{k=1, \cdots, a},\left(\eta_{1 a_{2 k+1}}^{*}, \eta_{2 a_{2 k+1}}^{*}\right)_{k=0, \cdots, b} .
\end{gathered}
$$


The antifields $\left(u_{a_{2 k}}^{*}, v_{a_{2 k}}^{*}\right)$ correspond to the Lagrange multipliers of the constraint functions $\bar{\gamma}_{a_{2 k}}$, respectively, $\gamma_{a_{2 k}}$, and $\left(u_{a_{2 k+1}}^{*}, v_{a_{2 k+1}}^{*}\right)$ are associated with $\gamma_{a_{2 k+1}}$, respectively, $\gamma_{a_{2 k+1}}^{\prime}$. The variables (119-120) have ghost number minus one, while in (121) there appear only ghost number minus two antifields. The gauge-fixing fermion should be taken to depend on the $\Phi^{A}$ 's, on the ghosts, and also on the antifields of the Lagrange multipliers, where

$$
\Phi^{A}=\left(q^{i}, p_{i}, y^{a_{k}}, \pi_{a_{k}}, z_{1}^{a_{2 k+1}}, p_{1 a_{2 k+1}}, z_{2}^{a_{2 k+1}}, p_{2 a_{2 k+1}}\right) .
$$

With the help of the gauge-fixing fermion we eliminate all the antifields except the antifields of the multipliers, and also the Lagrange multipliers. The gauge-fixed action will be expressed by

$$
\begin{aligned}
& S_{\psi}=\int d t\left(\dot{q}^{i} p_{i}+\sum_{k=1}^{L} \dot{y}^{a_{k}} \pi_{a_{k}}+\sum_{k=0}^{b}\left(\dot{z}_{1}^{a_{2 k+1}} p_{1 a_{2 k+1}}+\dot{z}_{2}^{a_{2 k+1}} p_{2 a_{2 k+1}}\right)+\right. \\
& \left.u_{a_{0}}^{*} \dot{\eta}^{a_{0}}+\sum_{k=1}^{L}\left(u_{a_{k}}^{*} \dot{\eta}_{1}^{a_{k}}+v_{a_{k}}^{*} \dot{\eta}_{2}^{a_{k}}\right)-H_{B}+[\psi, \Omega]\right)
\end{aligned}
$$

where the BRST charge and the BRST extension of the first-class Hamiltonian (116) respectively start like

$$
\begin{aligned}
& \Omega=\gamma_{a_{0}} \eta^{a_{0}}+\sum_{k=1}^{a}\left(\bar{\gamma}_{a_{2 k}} \eta_{1}^{a_{2 k}}+\gamma_{a_{2 k}} \eta_{2}^{a_{2 k}}\right)+ \\
& \sum_{k=0}^{b}\left(\gamma_{a_{2 k+1}} \eta_{1}^{a_{2 k+1}}+\gamma_{a_{2 k+1}}^{\prime} \eta_{2}^{a_{2 k+1}}\right)+\frac{1}{2} u_{a_{0}}^{*} \bar{C}_{b_{0} c_{0}}^{a_{0}} \eta^{b_{0}} \eta^{c_{0}}+ \\
& \quad \frac{1}{2} \sum_{j=1}^{a} \sum_{k=1}^{a} \sum_{i=1}^{a} v_{a_{2 j}}^{*} \bar{C}_{b_{2 k} c_{2 i}}^{a_{2 j}} \eta_{2}^{b_{2 k}} \eta_{2}^{c_{2 i}}+\cdots \\
& H_{B}=H_{0}^{\prime}+u_{a_{0}}^{*}\left(\tilde{V}_{b_{0}}^{a_{0}} \eta^{b_{0}}+Z_{a_{1}}^{a_{0}} \eta_{2}^{a_{1}}\right)+ \\
& \sum_{k=1}^{a} v_{a_{2 k}}^{*}\left(\eta_{1}^{a_{2 k}}+\sum_{j=1}^{a} \tilde{V}_{b_{2 j}}^{a_{2 k}} \eta_{2}^{b_{2 j}}+Z_{a_{2 k+1}}^{a_{2 k}} \eta_{2}^{a_{2 k+1}}+A_{a_{2 k-1}}^{a_{2 k}} \eta_{2}^{a_{2 k-1}}\right)+ \\
& v_{a_{1}}^{*}\left(\eta_{1}^{a_{1}}+A_{a_{0}}^{a_{1}} \eta^{a_{0}}+Z_{a_{2}}^{a_{1}} \eta_{2}^{a_{2}}\right)+ \\
& \sum_{k=1}^{b} v_{a_{2 k+1}}^{*}\left(\eta_{1}^{a_{2 k+1}}+A_{a_{2 k}}^{a_{2 k+1}} \eta^{a_{2 k}}+Z_{a_{2 k+2}}^{a_{2 k+1}} \eta_{2}^{a_{2 k+2}}\right)+\cdots
\end{aligned}
$$


The functions $\bar{C}_{b_{2 k} c_{2 i}}^{a_{2 j}}$ and $\tilde{V}_{b_{2 k}}^{a_{2 j}}$ are those involved with the irreducible gauge algebra arising in the $L$-stage reducible case. In this way, we realized the BRST quantization of arbitrary $L$-stage reducible first-class Hamiltonian systems in an irreducible manner, i.e., without introducing ghosts of ghosts. This completes our analysis.

\section{Example}

Here we exemplify the general theory exposed above in the case of abelian one- and two-form gauge fields with Stueckelberg coupling. We start with the Lagrangian action

$$
S_{0}^{L}\left[H^{\mu}, A^{\mu \nu}\right]=-\int d^{4} x\left(\frac{1}{12} F_{\mu \nu \rho}^{2}+\frac{1}{4}\left(M A_{\mu \nu}-F_{\mu \nu}\right)^{2}\right),
$$

where $F_{\mu \nu}$ and $F_{\mu \nu \rho}$ denote the field strengths associated with $H_{\mu}$, respectively, $A_{\mu \nu}$, and the notation $F_{\mu \nu \rho}^{2}$ signifies $F_{\mu \nu \rho} F^{\mu \nu \rho}$. (We used a similar notation for the other square.) The system described by action (126) possesses the first-class constraints

$$
\begin{gathered}
G_{i}^{(1)} \equiv \pi_{0 i} \approx 0, G^{(1)} \equiv \Pi_{0} \approx 0 \\
G_{i}^{(2)} \equiv-2 \partial^{l} \pi_{l i}+M \Pi_{i} \approx 0, G^{(2)} \equiv-\partial^{i} \Pi_{i} \approx 0,
\end{gathered}
$$

and the first-class Hamiltonian

$$
\begin{aligned}
& H=\int d^{3} x\left(-\pi_{i j}^{2}-\frac{1}{2} \Pi_{i}^{2}+A^{0 i} G_{i}^{(2)}+H^{0} G^{(2)}+\right. \\
& \left.\frac{1}{12} F_{i j k}^{2}+\frac{1}{4}\left(M A_{i j}-F_{i j}\right)^{2}\right) .
\end{aligned}
$$

In (127 129), the $\pi$ 's and $\Pi$ 's are the canonical momenta associated with the corresponding $A$ 's and $H$ 's. The first-class constraints (128) are first-stage reducible

$$
\partial^{i} G_{i}^{(2)}+M G^{(2)}=0
$$

with the reducibility functions

$$
Z_{a_{1}}^{a_{0}}=\left(\partial^{i}, M\right)
$$


The functions $A_{a_{0}}^{a_{1}} \mathrm{read}$

$$
A_{a_{0}}^{a_{1}}=\left(\begin{array}{c}
-\partial_{i} \\
-M
\end{array}\right),
$$

such that $Z_{a_{1}}^{a_{0}} A_{a_{0}}^{b_{1}}=-\left(\partial^{i} \partial_{i}+M^{2}\right)$ is invertible. The variables $\left(y^{a_{1}}, \pi_{a_{1}}\right)$ will be denoted in this case by $(\varphi, \pi)$. The irreducible first-class constraints are given by (127) and

$$
\gamma_{i}^{(2)} \equiv-2 \partial^{l} \pi_{l i}+M \Pi_{i}-\partial_{i} \pi \approx 0, \gamma^{(2)} \equiv-\partial^{i} \Pi_{i}-M \pi \approx 0,
$$

while the first-class Hamiltonian $H^{\prime}$ (see (54)) reads

$$
H^{\prime}=H+\int d^{3} x\left(-A^{0 i} \partial_{i} \pi-M H^{0} \pi\right) .
$$

We introduce the pairs $\left(\varphi_{1}, \pi_{1}\right),\left(\varphi_{2}, \pi_{2}\right)$, and set the constraints

$$
\gamma \equiv \pi-\pi_{1} \approx 0, \gamma^{\prime} \equiv \pi_{2} \approx 0 .
$$

The momentum $\pi$ is indeed a combination of the first-class constraints (133)

$$
\pi=-\frac{1}{\left(\partial^{i} \partial_{i}+M^{2}\right)}\left(\partial^{i} \gamma_{i}^{(2)}+M \gamma^{(2)}\right) .
$$

The first-class Hamiltonian with respect to (127), (133) and (135) has the form

$$
H_{0}=H^{\prime}+\int d^{3} x\left(-\varphi_{2}\left(\partial^{i} \gamma_{i}^{(2)}+M \gamma^{(2)}\right)-\varphi \pi_{2}\right) \equiv \int d^{3} x h_{0} .
$$

The extended action

$$
\begin{aligned}
& S_{0}^{E}=\int d^{4} x\left(\dot{A}^{0 i} \pi_{0 i}+\dot{A}^{i j} \pi_{i j}+\dot{H}^{0} \Pi_{0}+\dot{H}^{i} \Pi_{i}+\dot{\varphi} \pi+\dot{\varphi}_{1} \pi_{1}+\right. \\
& \left.\dot{\varphi}_{2} \pi_{2}-h_{0}-u^{i} G_{i}^{(1)}-u G^{(1)}-u^{\prime} \gamma-v^{i} \gamma_{i}^{(2)}-v \gamma^{(2)}-v^{\prime} \gamma^{\prime}\right),
\end{aligned}
$$

is invariant under the gauge transformations

$$
\begin{gathered}
\delta_{\epsilon} A^{0 i}=\epsilon_{1}^{i}, \delta_{\epsilon} H^{0}=\epsilon_{1}, \delta_{\epsilon} A^{i j}=\partial^{[i} \epsilon_{2}^{j]}, \delta_{\epsilon} H^{i}=\partial^{i} \epsilon_{2}+M \epsilon_{2}^{i}, \\
\delta_{\epsilon} \varphi=\partial_{i} \epsilon_{2}^{i}-M \epsilon_{2}+\tilde{\epsilon}_{1}, \delta_{\epsilon} \varphi_{1}=-\tilde{\epsilon}_{1}, \delta_{\epsilon} \varphi_{2}=\tilde{\epsilon}_{2}, \delta_{\epsilon} u=\dot{\epsilon}_{1}, \delta_{\epsilon} u^{\prime}=\dot{\tilde{\epsilon}}_{1}, \\
\delta_{\epsilon} v^{i}=\dot{\epsilon}_{2}^{i}-\partial^{i} \tilde{\epsilon}_{2}-\epsilon_{1}^{i}, \delta_{\epsilon} v=\dot{\epsilon}_{2}+M \tilde{\epsilon}_{2}-\epsilon_{1}, \delta_{\epsilon} v^{\prime}=\dot{\tilde{\epsilon}}_{2}+\partial_{i} \epsilon_{2}^{i}-M \epsilon_{2}+\tilde{\epsilon}_{1},
\end{gathered}
$$


the gauge variations of all the momenta being identically vanishing. In (139 141) the gauge parameters $\epsilon_{1}^{i}, \epsilon_{1}, \epsilon_{2}^{i}, \epsilon_{2}, \tilde{\epsilon}_{1}$ and $\tilde{\epsilon}_{2}$ are respectively associated with the constraint functions $G_{i}^{(1)}, G^{(1)}, \gamma_{i}^{(2)}, \gamma^{(2)}, \gamma$ and $\gamma^{\prime}$. From (139 141) we can derive the Lagrangian gauge transformations associated with the irreducible theory (including, of course, the gauge transformations of the original fields). In view of this we should consider a model of irreducible Hamiltonian theory. In this light we assume that (127) and the former constraint in (135) are primary, while (133) and the latter constraint from (135) are secondary. Passing from the extended action (138) to the corresponding total one (obtained by taking $v^{i}=0, v=0$ and $v^{\prime}=0$ in (138)) we derive its gauge invariances in the standard manner. Indeed, the equations $v^{i}=0, v=0$ and $v^{\prime}=0$ imply $\delta_{\epsilon} v^{i}=0, \delta_{\epsilon} v=0$ and $\delta_{\epsilon} v^{\prime}=0$. The last three equations lead via (141) to

$$
\epsilon_{1}^{i}=\dot{\epsilon}_{2}^{i}-\partial^{i} \tilde{\epsilon}_{2}, \epsilon_{1}=\dot{\epsilon}_{2}+M \tilde{\epsilon}_{2}, \tilde{\epsilon}_{1}=-\dot{\tilde{\epsilon}}_{2}-\partial_{i} \epsilon_{2}^{i}+M \epsilon_{2} .
$$

Replacing $\epsilon_{1}^{i}, \epsilon_{1}$ and $\tilde{\epsilon}_{1}$ from (142) in (139 140$)$ we get

$$
\begin{gathered}
\delta_{\epsilon} A^{0 i}=\dot{\epsilon}_{2}^{i}-\partial^{i} \tilde{\epsilon}_{2}, \delta_{\epsilon} H^{0}=\dot{\epsilon}_{2}+M \tilde{\epsilon}_{2}, \delta_{\epsilon} A^{i j}=\partial^{[i} \epsilon_{2}^{j]}, \delta_{\epsilon} H^{i}=\partial^{i} \epsilon_{2}+M \epsilon_{2}^{i}, \\
\delta_{\epsilon} \varphi=-\dot{\tilde{\epsilon}}_{2}, \delta_{\epsilon} \varphi_{1}=\dot{\tilde{\epsilon}}_{2}+\partial_{i} \epsilon_{2}^{i}-M \epsilon_{2}, \delta_{\epsilon} \varphi_{2}=\tilde{\epsilon}_{2} \\
\delta_{\epsilon} u=\ddot{\epsilon}_{2}+M \dot{\tilde{\epsilon}}_{2}, \delta_{\epsilon} u^{\prime}=-\ddot{\tilde{\epsilon}}_{2}-\partial_{i} \dot{\epsilon}_{2}^{i}+M \dot{\epsilon}_{2}
\end{gathered}
$$

The Lagrangian action corresponding to the above total action coincides with the original one, and its gauge transformations, which derive from (143 -144), read as

$$
\delta_{\epsilon} A^{\mu \nu}=\partial^{\mu} \epsilon^{\nu}-\partial^{\nu} \epsilon^{\mu}, \delta_{\epsilon} H^{\mu}=\partial^{\mu} \epsilon+M \epsilon^{\mu}, \delta_{\epsilon} \varphi_{1}=\partial_{\mu} \epsilon^{\mu}-M \epsilon .
$$

The gauge transformations for $\varphi$ and $\varphi_{2}$ were omitted as these fields play the role of Lagrange multipliers (see (137)) and are not relevant in the Lagrangian context. In order to write down (146) we employed the notations

$$
\epsilon^{\mu}=\left(\tilde{\epsilon}_{2}, \epsilon_{2}^{i}\right), \epsilon=\epsilon_{2} .
$$

In consequence, our formalism reproduces via (139 141) the original gauge transformations and outputs some new gauge transformations (for $\varphi_{1}$ ) that make the gauge transformation set (146) irreducible also at the Lagrangian 
level. The Lorentz covariance of the gauge transformations (146) is due to the introduction in the theory of the pairs $\left(\varphi_{1}, \pi_{1}\right)$ and $\left(\varphi_{2}, \pi_{2}\right)$.

In the sequel we approach the antifield BRST treatment of (138). Straightforward calculation then yield the solution to the master equation

$$
\begin{aligned}
& S^{E}=S_{0}^{E}+\int d^{4} x\left(A_{0 i}^{*} \eta_{1}^{i}+H_{0}^{*} \eta_{1}+A_{i j}^{*} \partial^{[i} \eta_{2}^{j]}+H_{i}^{*}\left(\partial^{i} \eta_{2}+M \eta_{2}^{i}\right)+\right. \\
& \varphi^{*}\left(\partial_{i} \eta_{2}^{i}-M \eta_{2}+\tilde{\eta}_{1}\right)-\varphi_{1}^{*} \tilde{\eta}_{1}+\varphi_{2}^{*} \tilde{\eta}_{2}+u_{i}^{*} \dot{\eta}_{1}^{i}+u^{*} \dot{\eta}_{1}+u^{*} \dot{\tilde{\eta}}_{1}+ \\
& v_{i}^{*}\left(\dot{\eta}_{2}^{i}-\partial^{i} \tilde{\eta}_{2}-\eta_{1}^{i}\right)+v^{*}\left(\dot{\eta}_{2}+M \tilde{\eta}_{2}-\eta_{1}\right)+ \\
& \left.v^{\prime *}\left(\dot{\tilde{\eta}}_{2}+\partial_{i} \eta_{2}^{i}-M \eta_{2}+\tilde{\eta}_{1}\right)\right) .
\end{aligned}
$$

All the ghosts from (148) have ghost number one, and all the antifields ghost number minus one. We take the gauge fixing fermion

$$
\begin{aligned}
& \psi=\int d^{4} x\left(u_{i}^{*}\left(\partial_{j} A^{j i}+M H^{i}+\partial^{i} \varphi_{1}\right)+u^{*}\left(\partial_{i} H^{i}-M \varphi_{1}\right)-\right. \\
& \left.u^{\prime *}\left(\partial_{j} A^{j 0}+M H^{0}\right)\right),
\end{aligned}
$$

which implements the irreducible gauge conditions $\partial_{j} A^{j i}+M H^{i}+\partial^{i} \varphi_{1}=0$, $\partial_{i} H^{i}-M \varphi_{1}=0$, and $\partial_{j} A^{j 0}+M H^{0}=0$. After some computation we are led to the gauge-fixed action

$$
\begin{aligned}
& S_{\psi}^{E}=S_{0}^{L}+\int d^{4} x\left(B_{\mu}\left(\partial_{\nu} A^{\nu \mu}+M H^{\mu}+\partial^{\mu} \varphi_{1}\right)+b\left(\partial_{\nu} H^{\nu}-M \varphi_{1}\right)+\right. \\
& \left.u_{\mu}^{*}\left(\square+M^{2}\right) \eta_{2}^{\mu}+u^{*}\left(\square+M^{2}\right) \eta_{2}\right),
\end{aligned}
$$

such that the resulting path integral is given by

$$
Z_{\psi}=\int \mathcal{D} A^{\nu \mu} \mathcal{D} H^{\mu} \mathcal{D} B_{\mu} \mathcal{D} \varphi_{1} \mathcal{D} u_{\mu}^{*} \mathcal{D} \eta_{2}^{\mu} \mathcal{D} u^{*} \mathcal{D} \eta_{2} \exp i S_{\psi}^{E} .
$$

In (150-151) we employed the identifications

$$
B_{\mu}=\left(\pi_{1}, \pi_{0 i}\right), b=\Pi_{0,} u_{\mu}^{*}=\left(-u^{\prime *}, u_{j}^{*}\right) \eta_{2}^{\mu}=\left(\tilde{\eta}_{2}, \eta_{2}^{j}\right) .
$$

One can check that there are no residual gauge invariances in action (150). Moreover, the gauge-fixed action (150) is Lorentz covariant. This is due precisely to the introduction in the theory of the pairs $\left(\varphi_{1}, \pi_{1}\right)$ and $\left(\varphi_{2}, \pi_{2}\right)$ subject to the constraints (135). 
While the gauge-fixing fermion (149) is useful in getting the covariant path integral (151), the fermion

$$
\begin{aligned}
& \psi^{\prime}=\int d^{4} x\left(u_{i}^{*}\left(\partial_{j} A^{j i}-\dot{A}^{0 i}+\partial^{i} \varphi_{1}\right)+\right. \\
& \left.u^{*}\left(\partial_{i} H^{i}-\dot{H}^{0}\right)-u^{*}\left(\partial_{j} A^{j 0}-\dot{\varphi}_{1}\right)\right)
\end{aligned}
$$

is appropriate in order to make the reduction to the physical degrees of freedom in the path integral. Starting with the solution (148) and on behalf of (153) we find after some computation the path integral over physical degrees of freedom for the model under consideration of the form

$$
\begin{aligned}
& Z_{\psi^{\prime}}=\int \mathcal{D} A^{i j} \mathcal{D} \pi_{i j} \mathcal{D} H^{i} \mathcal{D} \Pi_{i} \mathcal{D} \varphi_{1} \mathcal{D} \pi_{1} \delta\left(\partial^{j} \pi_{j i}+\partial_{i} \pi_{1}\right) \times \\
& \delta\left(\partial_{j} A^{j i}+\partial^{i} \varphi_{1}\right) \delta\left(\partial^{i} \Pi_{i}\right) \delta\left(\partial_{i} H^{i}\right) \exp i \bar{S}_{\psi^{\prime}}
\end{aligned}
$$

where $\bar{S}_{\psi^{\prime}}$ is given by

$$
\bar{S}_{\psi^{\prime}}=\int d^{4} x\left(\dot{A}^{i j} \pi_{i j}+\dot{H}^{i} \Pi_{i}+\pi_{i j}^{2}+\frac{1}{2} \Pi_{i}^{2}-\frac{1}{12} F_{i j k}^{2}-\frac{1}{4}\left(M A_{i j}-F_{i j}\right)^{2}\right) .
$$

The delta functions from the constraint functions and their gauge conditions in the path integral (154) show that the independent fields and momenta are precisely the transverse components of $H^{i}$ and $\Pi_{i}$ and also the longitudinal components of $A^{i j}$ and $\pi_{i j}$. It is clear that the conditions $\partial^{i} \Pi_{i}=0$ and $\partial_{i} H^{i}=$ 0 restrict the integration only over the two transverse degrees of freedom for the vector fields and their momenta (typically for electromagnetism). Related to the remaining conditions from the measure of (154), it can be shown that they enforce the longitudinal parts as independent components of the tensor fields and their momenta. Indeed, $A^{i j}$ and $\pi_{i j}$ can be decomposed into longitudinal and transverse components

$$
A_{i j}=\partial_{i} A_{j}^{T}-\partial_{j} A_{i}^{T}+\varepsilon_{i j k} \partial^{k} A^{L}, \pi_{i j}=\partial_{i} \pi_{j}^{T}-\partial_{j} \pi_{i}^{T}+\varepsilon_{i j k} \partial^{k} \pi^{L},
$$

where the transverse components satisfy $\partial^{i} A_{i}^{T}=0$ and $\partial^{i} \pi_{i}^{T}=0$. Then, the conditions $\partial_{j} A^{j i}+\partial^{i} \varphi_{1}=0$ and $\partial^{j} \pi_{j i}+\partial_{i} \pi_{1}=0$ imply via (156) that

$$
\partial^{i} \partial_{i} A_{j}^{T}+\partial_{j} \varphi_{1}=0, \partial^{i} \partial_{i} \pi_{j}^{T}+\partial_{j} \pi_{1}=0
$$


hence

$$
A_{j}^{T}=-\frac{\partial_{j}}{\triangle} \varphi_{1}, \pi_{j}^{T}=-\frac{\partial_{j}}{\triangle} \pi_{1}
$$

On the other hand, from (157) it follows that $\partial^{i} \partial_{i} \varphi_{1}=0$ and $\partial^{i} \partial_{i} \pi_{1}=0$, which then yield $\varphi_{1}=0, \pi_{1}=0$ by virtue of the boundary conditions for the unphysical degrees of freedom $\left(\varphi_{1}, \pi_{1}\right)$ (vacuum to vacuum). Inserting the last relations back in (158) we find that the conditions checked by the tensor fields and their momenta lead to $A_{j}^{T}=0$ and $\pi_{j}^{T}=0$, so the only physical degrees of freedom are described by the longitudinal pair $\left(A^{L}, \pi^{L}\right)$. In this way the conditions implemented in the measure of (154) lead to transverse degrees of freedom for the vector fields, respectively to a longitudinal one for the tensor fields, like in the reducible approach. This completes the analysis of the investigated model.

\section{Conclusion}

In conclusion, we succeeded in giving a systematic irreducible procedure of quantizing reducible first-class Hamiltonian systems accordingly the antifield BRST method. This new result was inferred by means of constructing an irreducible first-class Hamiltonian theory in a larger phase-space that remains physically equivalent to the original redundant one. The above equivalence makes legitimate the replacement of the quantization of the reducible theory by that of the irreducible system. As a consequence of our irreducible approach, the ghosts of ghosts, their antifields, as well as the pyramidal structure of auxiliary fields are no longer necessary. We further illustrate

in detail the theoretical part of the paper in the case of the Stueckelberg coupled abelian one- and two-form gauge fields.

\section{References}

[1] I.A. Batalin, G.A. Vilkovisky, Phys. Lett. B102 (1981) 27

[2] I.A. Batalin, G.A. Vilkovisky, Phys. Lett. B120 (1983) 166

[3] I.A. Batalin, G.A. Vilkovisky, Phys. Rev. D28 (1983) 2567 
[4] I.A. Batalin, G.A. Vilkovisky, J. Math. Phys. 26 (1985) 172

[5] M. Henneaux, Nucl. Phys. B (Proc. Suppl.) 18A (1990) 47

[6] M. Henneaux, C. Teitelboim, Quantization of Gauge Systems, Princeton Univ. Press, Princeton, 1992

[7] E.S. Fradkin, G.A. Vilkovisky, Phys. Lett. B55 (1975) 224

[8] I.A. Batalin, G.A. Vilkovisky, Phys. Lett. B69 (1977) 309

[9] E.S. Fradkin, T.E. Fradkina, Phys. Lett. B72 (1978) 343

[10] I.A. Batalin, E.S. Fradkin, Phys. Lett. B122 (1983) 157

[11] M. Henneaux, Phys. Rep. 126 (1985) 1

[12] J.M.L. Fisch, M. Henneaux, Phys. Lett. B226 (1989) 80

[13] J.M.L. Fisch, Mod. Phys. Lett. A5 (1990) 195

[14] R. Banerjee, J. Barcelos-Neto, Reducible constraints and phase-space extension in the canonical formalism, hep-th/9703020 